\title{
Transatlantica
}

Revue d'études américaines. American Studies Journal

\section{Benjamin Franklin, 1706-2008 : anatomie d'un tricentenaire transatlantique}

\section{Marie-Jeanne Rossignol}

\section{(2) OpenEdition}

1 Journals

\section{Édition électronique}

URL : https://journals.openedition.org/transatlantica/4431

DOI : 10.4000/transatlantica.4431

ISSN : $1765-2766$

Éditeur

Association française d'Etudes Américaines (AFEA)

\section{Référence électronique}

Marie-Jeanne Rossignol, « Benjamin Franklin, 1706-2008 : anatomie d'un tricentenaire

transatlantique », Transatlantica [En ligne], 2 | 2009, mis en ligne le 29 décembre 2009, consulté le 13 février 2023. URL : http://journals.openedition.org/transatlantica/4431 ; DOI : https://doi.org/10.4000/ transatlantica.4431

Ce document a été généré automatiquement le 13 février 2023.

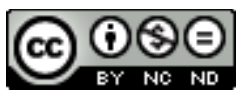

Creative Commons - Attribution - Pas d'Utilisation Commerciale - Pas de Modification 4.0 International - CC BY-NC-ND 4.0

https://creativecommons.org/licenses/by-nc-nd/4.0/ 


\title{
Benjamin Franklin, 1706-2008 : anatomie d'un tricentenaire transatlantique
}

\author{
Marie-Jeanne Rossignol
}

\section{Le tricentenaire aux Etats-Unis : commémorations et avancées historiographiques}

1 Les Pères fondateurs continuent, selon une tendance qui s'était renforcée depuis la fin des années 1990, à susciter une avalanche d'ouvrages, biographies en particulier, qui visent en partie le public universitaire, mais surtout un lectorat de masse éduqué. Certains auteurs, tels Joseph J. Ellis, font fortune grâce à la manne intellectuelle, politique et surtout mémorielle que constitue la période de création des Etats-Unis. Nous avions parlé de cette tendance avec Naomi Wulf dans un précédent dossier de Transatlantica (Wulf et Rossignol 2006). Il semblerait que le tricentenaire de la naissance de Benjamin Franklin (1706) ne soit pas demeuré en reste de cette vague de remémoration des «Fondateurs » : certains historiens professionnels ont même profité de l'occasion, comme me l'a signalé Naomi en relisant cette introduction, pour jeter un regard acéré sur l'ex - «bonhomme Franklin» dont l'image dans la conscience populaire s'est peut-être transformée par voie de conséquence, à moins que les critiques des historiens n'aient reflété la place nouvelle de Franklin dans l'imaginaire collectif états-unien.

2 Dès 2002, un historien célèbre, Edmund S. Morgan, publiait une biographie brève de Franklin (Morgan 2002). De nombreuses autres publications ont suivi: autres biographies brèves par des historiens connus comme Gordon S. Wood ou David Waldstreicher (Wood 2005, Waldstreicher 2006), ou par des auteurs de biographies à succès tels que Walter Isaacson ou Stacy Schiff (Isaacson, Schiff 2005), une biographie longue et savante de J. Leo Lemay qui n'a atteint que l'année 1757 et son troisième volume à l'heure où j'écris (Lemay 2008), des livres pour enfants, des rééditions de 
l'Autobiography, de The Way to Wealth, de Poor Richard's Almanack, des ouvrages consacrés à Franklin l'homme de science (Chaplin), Franklin le diplomate ... Il serait impossible de recenser toutes les publications d'ouvrages (1007 «produits» sur Amazon.com). D'autant que le tricentenaire de la naissance de Franklin suivait de près le bicentenaire de sa mort en 1990 qui avait déjà coïncidé avec la parution d'ouvrages dont un recueil d'essais édité par J. Leo Lemay (aussi éditeur des écrits dans la Library of America, Lemay 1997) : Reappraising Benjamin Franklin, qui avait rassemblé les signatures les plus expertes sur le sujet en 1993 (Lemay 1993). Un rapide coup d'œil à divers moteurs de recherche (Muse, Lion et Googlescholar) révèle que les publications recensées entre 2000 et 2008 sur Benjamin Franklin s'étagent entre 475 et 1474.

3 Deux expositions ont salué le tricentenaire de Benjamin Franklin. La Library Company de Philadelphie a mis l'accent sur Franklin auteur et imprimeur dans le cadre d'une exposition au catalogue magnifique organisée par James Green et Peter Stallybrass, alors que c'était le génie aux multiples facettes qui était présenté dans l'exposition organisée par Rosalind Remer et Page Talbott à l'initiative du Comité officiel du tricentenaire, exposition qui vint également s'installer à Paris lors de l'hiver 2007-2008, aux musées Carnavalet et des Arts et métiers (Talbott 2006). Ces deux expositions sont toujours accessibles par le biais de leurs sites web: http:// www.benjaminfranklin300.org/ pour la deuxième et http:// www.librarycompany.org/BFwriter/ pour la première. A Philadelphie, les autres événements, expositions et colloques, ateliers, ont été si nombreux qu'on ne saurait en rendre compte ici : notons seulement le colloque "The Atlantic World of Print» du McNeil Center for Early American History, du 28 au 30 septembre 2006; on peut également signaler que dès 2005, l'American Historical Association s'était réunie à Philadelphie pour y parler de Franklin (entre autres sujets) avant même le début des célébrations officielles, et que Rosalind Remer, déjà directrice exécutive du comité officiel des célébrations du tricentenaire de Franklin, avait fait partie du comité d'organisation du congrès sur place.

4 Ce tricentenaire a-t-il permis à des avancées historiographiques de se faire jour? Comment a-t-on présenté Benjamin Franklin? Il est difficile de ne pas admirer Franklin, doté de talents si divers; mais comme je l'ai suggéré plus haut, le tricentenaire ne s'est pas résumé à une série de manifestations et d'ouvrages hagiographiques. Même Edmund S. Morgan, dans sa biographie par ailleurs très favorable (Morgan 2002, 30), n'a pas caché que Franklin, l'homme de l'Autobiography, ne s'était jamais totalement livré : nous ne savons pas tout, en particulier sur sa vie privée. Le tricentenaire a donc permis aux critiques de Franklin de s'exprimer, comme David Waldstreicher qui, dans Runaway America, Benjamin Franklin, Slavery, and the American Revolution, a brisé la légende d'un Franklin antiesclavagiste (ce qu'il devint effectivement à la toute fin de sa vie) pour insister sur les ambiguïtés du personnage, plus typique des ambivalences de la Révolution américaine en matière de liberté et de libération que de ses vertus républicaines et démocratiques supposées (Waldstreicher).

David Waldstreicher n'a pas été le seul à remettre Franklin en question: plutôt que « first American » comme le prétend une autre biographie (Brands), Gordon S. Wood a dépeint Franklin comme le "dernier colonial» dans son excellent livre The Americanization of Benjamin Franklin (Wood 2004). Dans ce portrait paradoxal, Wood explique que Franklin s'est réinventé en Américain militant à partir de 1775, quand il a quitté précipitamment l'Angleterre ; mais il avait jusque-là mené une carrière de colon, 
et passé dix-huit ans en métropole. Ses années parisiennes (1776-1785) le comblèrent à tel point qu'il hésitait à rentrer, et à son retour, la nouvelle nation ne se montra pas particulièrement reconnaissante. Son petit-fils, par exemple, n'obtint jamais le poste diplomatique que son grand-père espérait. On voit donc, par le passage en revue de ces biographies marquantes, que le tricentenaire a permis d'écorner l'image d'un Franklin "Père de la nation ", figure archétypique de l'Américain aux manières simples, une image que Franklin lui-même avait largement travaillé à répandre en France, mais dont ses concitoyens (et surtout ses rivaux politiques) n'étaient pas dupes. Au fond, pour reprendre le titre d'un autre ouvrage d'Edmund S. Morgan paru à l'occasion du tricentenaire, Franklin fut "Not Your Usual Founding Father" (Wood 2006). Quant à Franklin scientifique, autre pôle de réflexion sans doute dû à la vogue de l'histoire des sciences, le livre de Joyce Chaplin (The First American. Benjamin Franklin and the Pursuit of Genius, 2006) fournit tous les éléments nécessaires à la compréhension de cette facette, elle aussi mythique, du personnage: mais la renommée scientifique ne fut-elle pas simplement pour Franklin le moyen d'accéder aux milieux sociaux et politiques qu'il ambitionnait d'atteindre? Tout en le suggérant, le livre de Chaplin ne va pas si loin dans la critique car la science resta un centre d'intérêt sincère de Franklin tout au long de sa vie.

\section{La venue de l'exposition Franklin à Paris : une commémoration opportune sur le plan diplomatique}

6 L'intérêt des commémorations n'est pas seulement d'ouvrir la voie de réinterprétations historiographiques: toute commémoration développe sa logique propre, et mérite d'être interprétée pour elle-même. Ce fut tout particulièrement le cas de ce tricentenaire: Franklin étant par essence un personnage transatlantique, qui avait laissé en France de son passage lors de la Guerre d'indépendance d'inoubliables traces dans la mémoire collective - qui lui valurent de vibrants éloges funèbres à sa mort en 1790, jusqu'à l'Assemblée nationale -, il était inévitable que se pose la question d'une commémoration en France, et cela à un moment où les relations entre les Etats-Unis et notre pays étaient au plus mal. Dès 2002, le Congrès avait établi une commission du tricentenaire, présidée par George Bush et composée de membres influents tels Joe Biden, actuel vice-président. Ce fut l'historienne Rosalind Remer, qui avait précédemment orchestré la création des programmes éducatifs du National Constitution Center à Philadelphie, et était l'auteur d'un livre sur les imprimeurs nordaméricains au XVIIIe siècle (Remer), à qui revint la mission de piloter les célébrations de 2004 à 2008, en conjonction avec un consortium d'institutions de Philadelphie, dont l'American Philosophical Society, et la Library Company, toutes deux fondées par Franklin, la Library Company (1 $1^{\text {ère }}$ bibliothèque de prêt aux Etats-Unis) en 1741 et l'APS en 1745. N'oublions pas l'Université de Pennsylvanie, qui fut elle aussi fondée par Franklin sous le nom de College of Philadelphia en 1755 et qui elle aussi adhéra au consortium (le Philadelphia Museum of Art et le Franklin Institute participèrent également) ${ }^{1}$.

7 Les discussions du transfert de l'exposition vers la France, engagées par Rosalind Remer avec Jean-David Levitte, alors ambassadeur à Washington, furent une des occasions du début de rapprochement diplomatique entre les deux nations après les heurts du déclenchement de la guerre contre l'Irak. On attribue couramment ce réchauffement des relations transatlantiques à $\mathrm{M}$. Levitte, qui devait par la suite devenir le premier 
directeur du Conseil de sécurité national français après l'élection de Nicolas Sarkozy à la présidence de la République : la venue de l'exposition Franklin à Paris en fut une des manifestations ${ }^{2}$. La préparation de la venue de l'exposition à Paris fut ponctuée de nombreuses réceptions, où des personnalités telles que Thierry de Montbrial et Gabriel de Broglie rappelèrent les grands moments de la carrière de Franklin, devant des parterres choisis, où les Sons et Daughters français de la Révolution américaine, comme d'autres groupes d'intérêt, trouvèrent l'occasion de démontrer leur engagement envers la cause de l'amitié franco-américaine ${ }^{3}$. Dès ce moment, et sachant que seul Thierry de Montbrial figurait comme universitaire dans la liste du comité français du tricentenaire (il y avait cependant plusieurs conservateurs de musée et directeurs d'institutions culturelles dont Jean-Noël Jeanneney), on pouvait craindre le manque d'authentiques et importants moments de coopération universitaire à l'occasion de cette commémoration.

8 En fait, l'arrivée de l'exposition Franklin à Paris s'accompagna surtout de quelques conférences destinées au grand public: après la conférence du 6 décembre 2007 de Joyce Chaplin au Musée des Arts et Métiers, organisée à l'initiative des Services culturels de l'Ambassade des Etats-Unis, la manifestation la plus scientifique, à ma connaissance, consista en une conférence à la Bibliothèque nationale le 17 janvier 2008 au soir, où officièrent Jim Green et Peter Stallybrass, historiens de la période coloniale, spécialistes de l'histoire du livre, responsables de l'exposition de la Library Company à Philadelphie et auteurs d'un catalogue remarquable (Green and Stallybrass). Ces incontestables spécialistes apportèrent brièvement en France le meilleur des connaissances sur l'histoire du livre en Amérique du nord. Pour le reste, André Kaspi donna une conférence le 31 janvier 2008 au musée des Arts et métiers (sur Franklin diplomate), où Thierry de Montbrial lui succéda le 6 mars (sur Franklin de la déclaration d'Indépendance à la Constitution). Au musée Carnavalet, ce fut Miriam Simon, conservatrice de l'exposition, qui consacra elle aussi une conférence à Franklin diplomate, thème obsédant, mais finalement assez révélateur de l'objectif principal de ce tricentenaire dans sa partie transatlantique : réconcilier l'Amérique et la France par le biais du souvenir de la Guerre d'indépendance, et de l'amitié franco-américaine que Franklin avait alors incarnée.

Rien d'étonnant à ce qu'un "comité français Benjamin Franklin 300 » ait été présidé par Laurence Chatel de Brancion, elle-même Daughter of the American Revolution, chapitre Rochambeau (chapitre français). Elle édita pour l'occasion une anthologie de textes de Franklin (Chatel de Brancion 2007), puis organisa un colloque dont les actes ont été édités récemment (Chatel de Bracion 2008). Docteur en histoire et spécialiste de Cambacérès, Laurence Chatel de Brancion a essayé d'aller au-delà des rencontres mondaines pour donner une dimension réellement scientifique aux manifestations qu'elle a organisées au nom du Comité Franklin, entre autres en invitant certains américanistes (historiens et anglicistes). On doit cependant regretter qu'elle n'ait pas su unir ses forces plus nettement à celles des spécialistes de la période coloniale, ou de Franklin lui-même. Leurs contributions à la commémoration, par des problématisations et des connaissances pointues, auraient pu ajouter aux manifestations « officielles » le piment de questionnements plus critiques, à l'instar de ce qui se passait aux Etats-Unis.

10 Si l'on excepte les publications coordonnées par Laurence Chatel de Brancion, malheureusement, loin de mettre en relief les publications récentes des spécialistes français de l'Amérique du nord, dont le livre de 2000 écrit par Nicole Fouché sur 
Franklin et Jefferson (Fouché), ou mieux encore, la biographie française de Franklin rédigée par Claude Fohlen, aussi publiée en 2000 (Fohlen), le tricentenaire "côté français » donna seulement, sur le plan éditorial, une certaine visibilité à une réédition du texte français de l'Autobiographie de Franklin, préfacée par l'astronome Jean Audouze (Audouze), alors que le catalogue français de l'exposition regroupait divers articles généraux ${ }^{4}$. Pourtant des publications françaises de qualité avaient marqué les commémorations du bicentenaire de la mort de Franklin, en 1990, et leurs auteurs auraient pu être mis à contribution 5 .

11 On doit cependant saluer le catalogue français de l'exposition qui constitue l'ouvrage le plus important consacré en France au tricentenaire de la naissance de Benjamin Franklin, et par voie de conséquence, saluer également le travail de muséographie qu'accomplirent les conservateurs impliqués en enrichissant considérablement l'exposition américaine (surtout au musée Carnavalet). Parmi les articles d'historiens et de politologues présents dans ce volume, on doit particulièrement noter l'article de Lucien Bély, historien spécialiste de la diplomatie européenne aux XVIIe et XVIIIe siècles, et véritable spécialiste de la Guerre d'indépendance ${ }^{6}$. C'est le seul article qui comprend une bibliographie extensive et bilingue, prenant en compte les travaux des spécialistes français de la Révolution américaine, tels Elise Marienstras ou Bernard Cottret. Pour le reste, ce bel ouvrage est typique de l'esprit de la commémoration: le titre renvoie à la comédie musicale de Vicente Minelli de 1951 (Benjamin Franklin: Un Américain à Paris) et place ainsi les deux expositions sur le mode de la légèreté plus que de la réflexion historique; le mot d'introduction de Bertrand Delanoë contient deux affirmations discutables (en seulement quelques lignes) ${ }^{7}$. Davantage que dans le questionnement historiographique, le plus grand intérêt de ce catalogue réside au final dans la présentation des objets que commentent avec érudition les conservateurs.

En guise de bilan, on doit donc signaler que nulle part les auteurs français d'ouvrages savants sur l'Amérique coloniale ou révolutionnaire, sur Benjamin Franklin lui-même, ne furent invités, consultés, à quelques rares exceptions près. Pour aller plus loin, cette commémoration a révélé une dichotomie entre les milieux de la diplomatie et de la culture et ceux de l'histoire savante, chacun ayant sa logique propre ; elle est donc passé à côté des débats qui agitaient la communauté historienne aux Etats-Unis et dont auraient pu rendre compte les américanistes français, sans parler de leur propre expertise et des points de vue originaux qu'ils auraient pu offrir ${ }^{8}$. Il est possible que quelque chose m'ait échappé, mais il se trouve que j'ai bien suivi les événements (paradoxalement grâce aux organisateurs américains), visité les expositions parisiennes, consulté leur catalogue et inspecté leurs boutiques. Sans croiser grandchose qui renvoie aux américanistes, historiens ou anglicistes, disposant d'un savoirfaire spécifique sur l'homme et la période. De même, les ouvrages importants parus aux Etats-Unis à l'occasion du tricentenaire ne furent pas traduits en français : seul The Great Improvisation de la biographe professionnelle Stacy Schiff (Schiff 2007), fit l'objet d'une traduction, probablement parce qu'il traitait exclusivement de Franklin diplomate à Paris. 


\section{Le colloque «Benjamin Franklin et les Lumières anglophones » en janvier 2008} politiques et culturelles, ont agencé cette commémoration transatlantique, en ignorant les seuls réels spécialistes de Franklin, et en insistant sur la médiatisation de l'ancienne amitié entre les deux nations, que Franklin incarna si brillamment? Après tout, le personnage de Benjamin Franklin, architecte entre 1779 et 1784 d'une bonne entente entre les deux nations, se prêtait parfaitement à une telle instrumentalisation. Le recours à de grands noms (Montbrial, Kaspi) garantissait l'oreille du public le plus large. De même, les conférences sur des thématiques très généralistes. D'autres grandes personnalités du monde universitaire qui ont participé au catalogue français de l'exposition Franklin, tels Daniel Roche, ont pu également rassurer un public éduqué et le conforter dans ses connaissances, mais sans lui offrir une ouverture sur de nouvelles problématiques, ce qui aurait pu être le cas si l'objectif de la venue de l'exposition Franklin à Paris avait été de faire découvrir l'homme, son temps et son pays (finalement la jeune République américaine) au public français. parties à Paris, et renforçait plutôt l'imagerie traditionnelle de Franklin en France: Benjamin Franklin le scientifique (une image chère aux Européens depuis le XVIIIe siècle) trouva asile au musée des Arts et métiers, tandis que Franklin l'homme politique et surtout le diplomate, était hébergé au musée Carnavalet où de nombreux apports des collections françaises permettaient de mettre encore mieux en valeur que dans l'exposition originale l'importance de la Guerre d'indépendance et la figure d'amitié transatlantique que fut Franklin.

Il eût donc été donc dommage de ne pas profiter de la venue à Paris de cette exposition, et d'une certaine agitation médiatique parisienne autour du personnage de Benjamin Franklin, pour mettre en valeur les spécialistes français du célèbre scientifique, imprimeur et diplomate, ne serait-ce qu'au sein de notre propre communauté. Et pour parler de Franklin sous toutes ses facettes à l'occasion du tricentenaire, sans se limiter à ses activités de diplomate à Paris, même si elles ne pouvaient être ignorées. Alors que sous la plume des historiens américains, Franklin apparaissait plus que jamais comme un «colonial» ambitieux, un savant cosmopolite qui s'était considéré avant tout comme britannique jusqu'en 1775, il semblait utile d'inviter un collègue britanniste pour parler de Franklin dans le cadre de la pensée anglophone du XVIIIe siècle (Robert Mankin). D'où l'idée d'un colloque sur «Benjamin Franklin et les Lumières anglophones" permettant à nos collègues, américanistes et anglicistes, de faire partager leur recherche sur Franklin et son époque, en universitaires et savants, devant un public ouvert également aux étudiants.

Se joignirent aux spécialistes français Elise Marienstras et Christian Lerat (auteur d'une thèse d'Etat marquante sur Franklin, Lerat 1985), deux collègues anglophones Michael Zuckermann et James Delbourgo, le premier spécialiste de l'Amérique pré-industrielle (Zuckerman), et auteur de nombreux articles sur Franklin; le second spécialiste d'histoire des sciences (Delbourgo et Delbourgo et Dew). Nathalie Caron et Naomi Wulf acceptèrent, à cette occasion, de poursuivre la réflexion sur les «Lumières américaines » qu'elles avaient entamée en 2002 dans un numéro de la Revue Française d'Etudes Américaines. Claire Parfait, Jim Green et Rosalind Remer participèrent à cette

Transatlantica, 2 | 2009 
occasion à une table ronde sur l'histoire du livre en Amérique du nord à l'époque de Franklin. Une exposition de photos contemporaines des lieux où vécut Franklin en France et en Amérique du nord (Boston, Philadelphie, Paris) ornait la salle du colloque : j'ai sélectionné mes photos préférées des représentations de Franklin à Paris et Philadelphie pour illustrer ce dossier, mais Noëlle de Chambrun avait également participé à cette exposition (photos de Boston).

Le dossier qui suit reprend l'essentiel des communications originales présentées le 18 janvier 2008: l'angle critique propre aux commémorations nord-américaines s'y retrouve, en particulier sous la plume de Michael Zuckerman, qui dépeint un Franklin opportuniste et s'interroge sur les ressorts véritables d'une identité américaine peutêtre sans substance; mais les contributeurs français ont aussi apporté leur regard original.

Se détachant nettement d'une vision angélique de l'«amitié franco-américaine», Christian Lerat s'est penché sur les deux années (un peu moins en fait, de décembre 1776 à février 1778) qui séparent l'arrivée de Franklin à Paris de la signature des traités franco-américains de 1778 : rien n'était moins sûr que cette alliance improbable et il a fallu toutes les qualités "florentines" d'un Franklin pour convaincre la monarchie française d'aider une jeune République. Ainsi ce spécialiste du savant et homme politique américain a-t-il dévoilé les ressorts géopolitiques et humains d'une " amitié » artificielle, bien loin de l'exaltation décrite par Stacy Schiff dans son livre sur le séjour de Franklin à Paris.

De toutes les facettes de son personnage, c'est peut-être le Franklin populaire, le "Bonhomme Richard», qui a assuré pendant deux siècles la notoriété de Benjamin Franklin, comme en attestait sa présence sur tous les timbres postes, avec George Washington. Mais les timbres du tricentenaire, nous explique François Brunet, ont définitivement substitué à cette imagerie le personnage du savant et de l'intellectuel.

Un savant qui de son vivant n'avait d'ailleurs pas toujours été l'objet d'une admiration aussi totale que le prétend la légende: Franklin colonial, bourgeois, pragmatiste, ne pouvait recevoir d'éloges de ceux qui considéraient que la science se devait d'être métropolitaine, aristocratique et fondée sur des lois mathématiques, nous explique James Delbourgo dans un article qui joint à l'analyse épistémologique le contexte impérial et social dans un cadre atlantique.

21 L'article d'Elise Marientras, sans nier le pragmatisme de Franklin, voit en lui le maître du temps des Lumières, qu'il incarna remarquablement, tant sa confiance dans le progrès de l'humanité et de sa nation était prégnante et s'appliquait à toutes les facettes de la vie.

Mais, nous rappelle James Delbourgo, les Lumières coloniales n'étaient pas toujours vues d'un oeil favorable sur le continent, surtout au moment de la révolution américaine, où l'on ne peut distinguer les querelles épistémologiques de conflits politiques et sociaux. L'article de Robert Mankin illustre bien le lien entre science et politique impériale à travers un écrit scientifique de Franklin: le savant ne peut s'empêcher, en décrivant le polype dans un article de 1755, d'en tirer des conclusions pour les nations. A travers leurs colonies, celles-ci peuvent selon lui produire d'autres nations, pour peu que le terreau d'implantation soit propice. Des propos séduisants pour l'empire britannique, et que l'on retrouve plus tard sous la plume de Hume ; mais ils annonçaient également la possibilité de la future autonomie des colonies! 
Nathalie Caron et Naomi Wulf reviennent pour leur part en détail sur la problématique plus large des "Lumières américaines ", un thème qu'elles avaient exploré une première fois en 2002 dans un dossier de la Revue française d'études américaines. Elles constatent depuis cette date une vague de publications sur le sujet. Face à cet apparent renouveau historiographique, elles repèrent cependant certaines tentatives d'instrumentalisation de la notion: les "néo-conservateurs " cherchent par ce biais à renouer avec l'habituel débat qui opposerait une "bonne " Révolution américaine éclairée, à la Révolution française ; le rôle de la religion prête aussi à débats, tenants de Lumières chrétiennes ou déistes s'opposant, au prisme de controverses contemporaines.

\section{BIBLIOGRAPHIE}

AUDOUZE, Jean, « Moi, Benjamin Franklin, citoyen du monde, homme des Lumières » : autobiographie et textes scientifiques de Benjamin Franklin réunis et commentés par Jean Audouze. Ed. Dunod, mai 2006

BRANDS, H.W. The First American : the Life and Times of Benjamin Franklin. New York: Doubleday, 2000.

CARON, Nathalie, et Naomi WULF. «Introduction : Les lumières américaines dans l'historiographie contemporaine aux Etats-Unis : ambivalences et réticences », Revue française d'études américaines, Les lumières américaines, 92, 2002 (2), pp.3-21.

CHAPLIN, Joyce. The First Scientific American. Benjamin Franklin and the Pursuit of Science. New York : Basic Books, 2006.

CHATEL DE BRANCION, Laurence. Benjamin Franklin à la recherche d'un monde meilleur. Paris : Economica, 2007.

---, et Ellen HAMPTON eds. La Naissance des Etats-Unis d'Amérique a-t-elle tué l'Europe des Lumières? Actes du colloque, Paris : Editions SPM, 2008.

COTTRET, Bernard. La Révolution américaine. La quête du bonheur. Paris : Perrin, 2003.

DELBOURGO, James. A Most Amazing Scene of Wonders: Electricity and

Enlightenment in Early America.Cambridge, Mass.: Harvard University Press, 2006.

---, and Nicholas DEW eds. Science and Empire in the Atlantic World. London ; New York : Routledge, 2007.

GREEN, James N., et Peter STALLYBRASS. Benjamin Franklin: Writer and Printer. New Castle, Del.: Oak Knoll Press, the Library Company of Philadelphia, and the British Library, 2006.

FOHLEN, Claude. Benjamin Franklin : l'Américain des Lumières. Paris : Payot \& Rivages, 2000.

FOUCHE, Nicole. Benjamin Franklin \& Thomas Jefferson : aux sources de l'amitié franco-américaine, 1776-1808; préf. par Claude Folhen. Paris : M. Houdiard, 2000. 
HUGUES, Gérard et Daniel ROYOT, eds. "Benjamin Franklin : Des Lumières à nos jours”, Etudes anglaises, 95 (1991).

ISAACSON, Walter. Benjamin Franklin : An American Life. New York : Simon and Schuster, 2003.

LEMAY, J. Leo. Autobiography, Poor Richard, and later writings : letters from London, 1757-1775, Paris 1776-1785, Philadelphia 1785-1790. New York : Library of America, 1997.

---. Reappraising Benjamin Franklin. Newark : University of Delaware Press ; London ; Cranbury, NJ : Associated University Presses, 1993.

---.The Life of Benjamin Franklin: Soldier, Scientist, and Politician, 1748-1757, Philadelphia: University of Philadelphia Press, 2008.

LERAT, Christian. Benjamin Franklin, Philosophe de l'Ordre et de la Liberté, Thèse de doctorat d'État, Bordeaux, 1985, 6 vol.

---. Benjamin Franklin : quand l'Amérique s'émancipait. Talence : Presses universitaires de Bordeaux, 1992.

MARIENSTRAS, Elise. Les Mythes fondateurs de la nation américaine : essai sur le discours idéologique aux États-Unis à l'époque de l'indépendance, 1763-1800. (1976) Paris : Editions complexes, 1992.

---. « Nous, le peuple » : les origines du nationalisme américain. Paris : Gallimard, 1988.

MORGAN, Edmund S. Benjamin Franklin. New Haven and London : Yale University Press, 2002.

---. Not Your Usual Founding Father. Selected Readings from Benjamin Franklin. New Haven and London: Yale University Press, 2006.

Musée Carnavalet et musée national des techniques. Benjamin Franklin. Un Américain à Paris (1776-1785): 5 décembre 2007-9 mars 2008. [catalogue de l'exposition du musée Carnavalet). Paris: Paris Musées, 2007.

REMER, Rosalind. Printers and Men of Capital: Philadelphia Book Publishers in the New Republic. Philadelphia: Univ. of Pennsylvania Pr., 1996.

SCHIFF, Stacy. The Great Improvisation : Benjamin Franklin, France and the Birth of the United States. Henry Holt, 2005.

---. La grande improvisation: Benjamin Franklin, la France et la naissance des Etats-Unis. Paris : Grasset, 2007

TALBOTT, Page. Benjamin Franklin: In Search of a Better World. New Haven: Yale University Press, 2005.

WALDSTREICHER, David. Runaway America, Benjamin Franklin, Slavery, and the American Revolution. New York : Hill and Wang, 2005.

WOOD, Gordon S. The Americanization of Benjamin Franklin. New York : Penguin Press, 2004.

WULF, Naomi, avec le concours de Marie-Jeanne ROSSIGNOL. Transatlantica 2006:2 Révolution « Dossier: Autour de la Révolution américaine », «Introduction ».

ZUCKERMAN, Michael. « Doing Good While Doing Well: Benevolence and Self-Interest in Franklin's Autobiography, » in J. A. Leo Lemay, ed., Reappraising Benjamin Franklin: A Bicentennial Perspective. Newark, DE. : University of Delaware Press, 1993., pp. 441-51.

---. « The Selling of the Self: From Franklin to Barnum, » in Barbara Oberg and Harry Stout, eds., Benjamin Franklin, JonathanEdwards, and the Representation of American Culture. Oxford: Oxford University Press, 1993. pp. 152-67. 
---. « An Inclination Joined with an Ability to Serve, » in Peter Conn, ed., The Autobiography of Benjamin Franklin. Philadelphia, University of Pennsylvania Press, 2005. pp. 154-8.

---. « Food for Thought: America's Accessible Founder ». Pennsylvania Legacies 6:1 (May, 2006). pp. 36-37.

---. « Benjamin Franklin at 300: The Show Goes on. A Review of the Reviews ». Pennsylvania Magazine of History and Biography 131(2007). pp. 181-211.

---. « Founding Fathers: Franklin, Jefferson, and the Educability of Americans, » in John Pollack and Michael Ryan, eds., Educatingthe Youth of Pennsylvania: Worlds of Learning in the Age ofFranklin. Philadelphia, Van Pelt Library, 2006.

\section{NOTES}

1. Depuis Ros Remer et Page Talbott, fortes du succès de l'organisation du tricentenaire, ont fondé une société Remer \& Talbott, pour offrir leurs services en ingénierie historique aux institutions et aux personnes et entreprises privées. Pour les détails de ce que j'entends par «l'ingénierie historique », voir leur site : www.remertalbott.com/

2. Rosalind Remer m'a personnellement expliqué comment la venue de l'exposition à Paris fut l'objet de discussions directes entre elle-même et l'ambassadeur de France Levitte à Washington. Ce qui est confirmé par Page Talbott dans le catalogue de l'exposition Carnavalet : « Le Benjamin Franklin Tercentenary tient à remercier les trois ambassadeurs qui ont soutenu ces projets renforçant l'amitié franco-américaine, S.E. Jean-David Levitte, ancien ambassadeur de France aux Etats-Unis, S.S Howard Leach, ancien ambassadeur des Etats-Unis en France, et S.E Craig R. Stapleton, ambassadeur des Etats-Unis en France. » (Musées, 11).

3. Le 3 juillet 2006 fut ainsi par exemple organisée une visite du château de Breteuil, en présence du marquis et de la marquise, de l'ambassadeur des Etats-Unis, pour un public sélectionné (dont le comité américain du tricentenaire), devant lequel Gabriel de Broglie présenta les qualités de diplomate de Benjamin Franklin. Pour la liste des soutiens officiels français au tricentenaire, voir le site http://www.benfranklin.org.honorary.htm/

4. Catalogue de l'exposition: Benjamin Franklin: un Américain à Paris, 1776-1785: 5 décembre 2007-9 mars 2008, Musée Carnavalet-Histoire de Paris / [textes de Lucien Bély, Jean-Claude Bonnet, Renée Davray-Piékolek, et al. Paris : Paris musées, impr. 2007

5. En particulier un numéro spécial de la revue Etudes anglaises $(95,1991)$ paru en 1991 « Benjamin Franklin: Des Lumières à nos jours ». Edité par Gérard Hugues et Daniel Royot, il comprenait aussi une contribution de Christian Lerat, entre autres. Merci à $C$. Lerat de m'avoir indiqué que ce volume avait suivi la tenue d'un important colloque à Lyon.

6. Lucien Bély, historien de renom, est l'auteur d'une dizaine d'ouvrages sur l'histoire de la diplomatie, dont L'art de la paix en Europe. Naissance de la diplomatie moderne XVI $-\mathrm{XVIII}^{e}$ siècles, PUF, 2007.

7. B. Delanoë écrit «Cet homme, qui a libéré ses esclaves, devint un grand ami de Voltaire» (Musée Carnavalet et musée national des techniques, 9). Mais pour David Waldstreicher, Franklin continua pendant son séjour parisien à protéger les maîtres et non leurs esclaves. Et son dernier esclave ne fut pas libéré, mais mourut à Philadelphie en 1781 (Waldstreicher, 221-230). Quant à l'amitié entre Franklin et Voltaire, elle consista surtout en une rencontre très médiatisée entre ces deux grands personnages des Lumières.

8. A part la thèse de 1985 soutenue par Christian Lerat, les thèses récentes sur Benjamin Franklin, en histoire ou en études américaines, ne semblent pas avoir donné lieu à publication. Et leurs auteures n'ont pas plus été appelées à éclairer les commémorations de leurs connaissances. Il 
s'agit de Marie-Hélène Naquet, Benjamin Franklin: Image et Influence en France. De L'actualité à la postérité (1776-1830), dirigée par Daniel Royot à Paris 3 et soutenue en 1997, et de Le Bonhomme Richard de Benjamin Franklin à la conquête de l'Europe : La diffusion d'un bestseller américain en France, en Grande-Bretagne et dans les Etats allemands des années 1770 à 1830, dirigée par Daniel Roche à Paris 1 et soutenue en 1999.

\section{RÉSUMÉS}

Cet article analyse le Tricentenaire de Benjamin Franklin, qui eut lieu de 2006 à 2008. A l'occasion de cette date anniversaire, de nombreux livres furent publiés, des colloques et expositions organisés aux Etats-Unis. Ces travaux et manifestations ont produit un nouveau regard, critique, sur Franklin, père fondateur assez extraordinaire. L'exposition principale est venue à Paris, au cours de l'hiver 2007-2008, clairement dans le cadre d'une volonté de rapprocher les deux pays à la suite des controverses suscitées par la Guerre en Irak. Cette commémoration francoaméricaine a eu comme objectif principal de souligner la diplomatie et la collaboration transatlantiques, et non de présenter Franklin sous toutes ses facettes, et de l'intégrer au contexte de son époque dans son pays. Un symposium fut donc organisé à l'Université Paris Diderot afin de mettre en valeur les travaux scientifiques sur Franklin, français, mais aussi étrangers. Les principales communications sont présentées dans ce dossier.

This paper offers reflections on the Benjamin Franklin Tercentenary, which took place from 2006 to 2008. This anniversary was celebrated by many publications, conferences and exhibitions in the United States, producing a renewed and critical understanding of Franklin, "not your usual founding father". The main exhibition travelled to France (2007-2008), clearly as part of an attempt to patch up relationships between the two countries in the wake of the Iraq war controversy. The main focus in this Franco-American commemoration was on transatlantic diplomacy and collaboration, not really on Franklin in his multiple capacities, in his time and country of birth. The conference organized at Université Paris-Diderot in January 2008 was by contrast a scholarly affair, highlighting work on Franklin conducted by French and foreign specialists. Most of the papers are presented here.

\section{INDEX}

Keywords : Benjamin Franklin, Founding Fathers, transatlantic commemoration

Mots-clés : Benjamin Franklin, commémoration transatlantique, Pères fondateurs

\section{AUTEUR}

\section{MARIE-JEANNE ROSSIGNOL}

Université Paris -Diderot

rossignol@univ-paris-diderot.fr

Marie-Jeanne Rossignol est Professeur de civilisation américaine à l'Université Paris-Diderot. Elle a publié en 1994 Le ferment nationaliste : aux origines de la politique extérieure des Etats-Unis 1789-1812 
(Paris : Belin), puis coordonné en 2005 la publication du numéro 95 des Cahiers Charles $V$ : «L'Amérique : des colonies aux républiques » (avec Lucia Bergamasco). Elle vient de publier avec Jacques Portes, Cécile Vidal et Nicole Fouché Europe / Amérique du nord. Cinq siècles d'interactions (Paris : Armand Colin, 2008) et prépare un livre sur le premier mouvement antiesclavagiste aux Etats-Unis. 